\title{
Study Of Boosted W-Jets And Higgs-Jets With the SiFCC Detector
}

\author{
Shin-Shan Yu*, Sergei Chekanov, ${ }^{b}$ Lindsey Gray, ${ }^{c}$ Ashutosh Kotwal, ${ }^{c, d}$ \\ Sourav Sen, ${ }^{d}$ and Nhan Viet $\operatorname{Tran}^{c}$ \\ ${ }^{a}$ Department of Physics, National Central University, Chung-Li, Taiwan \\ ${ }^{b}$ High Energy Physics Division, Argonne National Laboratory, Argonne IL, USA \\ ${ }^{c}$ Fermi National Accelerator Laboratory, Batavia, USA \\ ${ }^{d}$ Department of Physics, Duke University, Durham NC, USA \\ E-mail: syu@cern. ch, chekanoveanl.gov, lagrayefnal.gov, \\ kotwal@phy . duke. edu, ss 567 @phy. duke. edu, ntran@fnal.gov
}

\begin{abstract}
We study the detector performance in the reconstruction of hadronically-decaying $\mathrm{W}$ bosons and Higgs bosons at very high energy proton colliders using a full GEANT4 simulation of the SiFCC detector. The $\mathrm{W}$ and Higgs bosons carry transverse momentum in the multi-TeV range, which results in collimated decay products that are reconstructed as a single jet. We present a measurement of the energy response and resolution of boosted W-jets and Higgs-jets and show the separation of two sub-jets within the boosted boson jet.
\end{abstract}

38th International Conference on High Energy Physics 3-10 August 2016

Chicago, USA

\footnotetext{
* Speaker.
} 


\section{Introduction}

A very high energy proton-proton collider in the future, such as FCC-hh at $\sqrt{s}=100 \mathrm{TeV}$ [1] or SPPC at $\sqrt{s}=70 \mathrm{TeV}[2,3]$, will set many challenges for the detector design. High-energy $p p$ colliders can produce particles with large mass, giving decay products with large transverse momentum. For searches or measurements that involve hadronic final states, the response and resolution of jets are major sources of systematic uncertainties in the analysis. In order to determine precisely the energy of a boosted jet, a calorimeter for future $p p$ colliders must satisfy the following requirements: (i) good containment up to $p_{\mathrm{T}}(\mathrm{jet}) \approx 30 \mathrm{TeV}$, which implies a total interaction length of $\approx 12 \lambda_{\mathrm{I}}$ for the full calorimeter (electromagnetic and hadronic) [4]; (ii) small constant term in the energy resolution of hadron calorimeter: $c<3 \%$, since the constant term dominates jet energy resolution for $p_{\mathrm{T}}(\mathrm{jet})>5 \mathrm{TeV}$; (iii) sufficient transverse segmentation for resolving boosted particles; and (iv) longitudinal segmentation for detailed information on the shower profile. A good starting point and a promising approach for high energy $p p$ colliders, aiming to satisfy the requirements listed above, can be a detector based on the Silicon Detector ( $\mathrm{SiD}$ ) concept [5] developed for the International Linear Collider [6, 7]. The name of this detector is "SiFCC", where "Si" indicates that it shares several design features of the all-silicon SiD detector. This document studies the performance of the SiFCC detector using a full GEANT4 simulation and the physics events containing boosted W-jets and Higgs-jets. The studies shown in this document focus on the performance of the electromagnetic and hadron calorimeters of the SiFCC detector.

\section{Description of the SiFCC Detector}

As with most of the multi-purpose detectors, the SiFCC detector has a cylindrical shape. The diameter and the length of the SiFCC detector are $18 \mathrm{~m}$ and $20.1 \mathrm{~m}$, respectively. The size of the SiFCC detector is smaller than the ATLAS detector but larger than the CMS detector. The version of the SiFCC detector for the study in this document is version 7, currently with the focus on the barrel region. The central feature of the SiFCC detector is a superconducting solenoid, of $4.8 \mathrm{~m}$ internal diameter, providing an axial magnetic field of 5 Tesla along the beam direction. Within the superconducting solenoid volume are a silicon pixel and strip tracker, a silicon-tungsten electromagnetic calorimeter (ECAL), and a scintillator-steel hadron calorimeter (HCAL). The silicon pixel detector has a readout pitch size of $20 \mu \mathrm{m}$, with 5 layers in the barrel and 4 disks in each endcap. The silicon strip detector has a readout pitch size of $50 \mu \mathrm{m}$, with 5 layers in the barrel and 4 disks in each endcap. The ECAL, which surrounds the tracker volume, is finely-segmented, and has a cell size of $2 \mathrm{~cm} \times 2 \mathrm{~cm}, 32$ longitudinal layers, and a total radiation length of $\approx 35 X_{0}$. The HCAL cell size is $5 \mathrm{~cm} \times 5 \mathrm{~cm}$, with 64 longitudinal layers, and a total interaction length of $\approx 11.25 \lambda_{\mathrm{I}}$ to provide good containment for single hadrons with energies above $1 \mathrm{TeV}$ and a $p_{\mathrm{T}}=$ $40 \mathrm{TeV}$ jet [4].

\section{Simulated Samples}

In order to study the performance of the SiFCC detector, we use three different sets of simulated samples: (i) single pion, (ii) $\mathrm{Z}^{\prime} \rightarrow \mathrm{WW} \rightarrow \mathrm{q} \overline{\mathrm{q}}^{\prime} \mathrm{q}^{\prime} \overline{\mathrm{q}}$, and (iii) $\mathrm{H}_{2} \rightarrow \mathrm{hh} \rightarrow \mathrm{b} \bar{b} \mathrm{~b} \overline{\mathrm{b}}$ in the $2 \mathrm{HDM}$ 
model. The single pion samples are generated using the ProMC package [8]. Pions in this sample are produced at the origin $(x, y, z)=(0,0,0)$ with a uniform distribution in $\phi=0-2 \pi$ and $|\eta|<3.5$ and the energy of the pion is $2^{n} \mathrm{GeV}$ for $n=1-15$. Given that our focus is on the detector performance, in order to eliminate the need of reconstructing particles from the underlying event present in $p p$ collisions, both the $\mathrm{Z}^{\prime}$ and the $\mathrm{H}_{2}$ are produced in $\mu^{+} \mu^{-}$collisions. The $\mathrm{Z}^{\prime}$ samples are generated with PYTHIA 6 [9] in $\mu^{+} \mu^{-}$collisions for $\sqrt{s}=M_{Z^{\prime}}=5,10,20$, and $40 \mathrm{TeV}$, respectively. The $2 \mathrm{HDM} \mathrm{H}_{2}$ sample is generated at LO with MADGRAPH5_AMC @ NLO 5.2.3.3 [10] in $\mu^{+} \mu^{-}$ collisions at $\sqrt{s}=5 \mathrm{TeV}$ for $M_{\mathrm{H}_{2}}=5 \mathrm{TeV}$; the parton showering and hadronization are performed with PYTHIA 6. All samples are processed through a GEANT4-based [11] simulation of the SiFCC detector version 7. The simulated samples are provided by the HepSim repository [12]. and the computations were performed using the Open-Science grid [13].

\section{Energy Response and Resolution for Single Pions}

We study the energy response and resolution of SiFCC calorimeters using the single pion samples as described in Section 3. The $|\eta|$ of the pion must be less than 1.1. The energy response is evaluated by taking the $90 \%$ truncated mean of the energy clustered with calorimeter hits and dividing it with the true pion energy, while the resolution is the ratio of the $90 \%$ truncated RMS to the $90 \%$ truncated mean. The clustering is performed with the anti-kt algorithm with a distance parameter of 0.4 [14], as implemented in the FASTJET package [15]. The calorimeter energy response and resolution in Fig. 1 are fitted with the functions below:

$$
y_{\text {response }}=A+B \cdot \log \left(E_{\text {true }}\right), \quad y_{\text {resolution }}=\frac{0.43}{\sqrt{E_{\text {true }}}} \oplus 0.009 .
$$

where $A=0.7,0.86,1$, and $B=0.077,0.0202,0$ for $E_{\text {true }}$ in the range of 2-16, 16-2000, and above $2000 \mathrm{GeV}$.
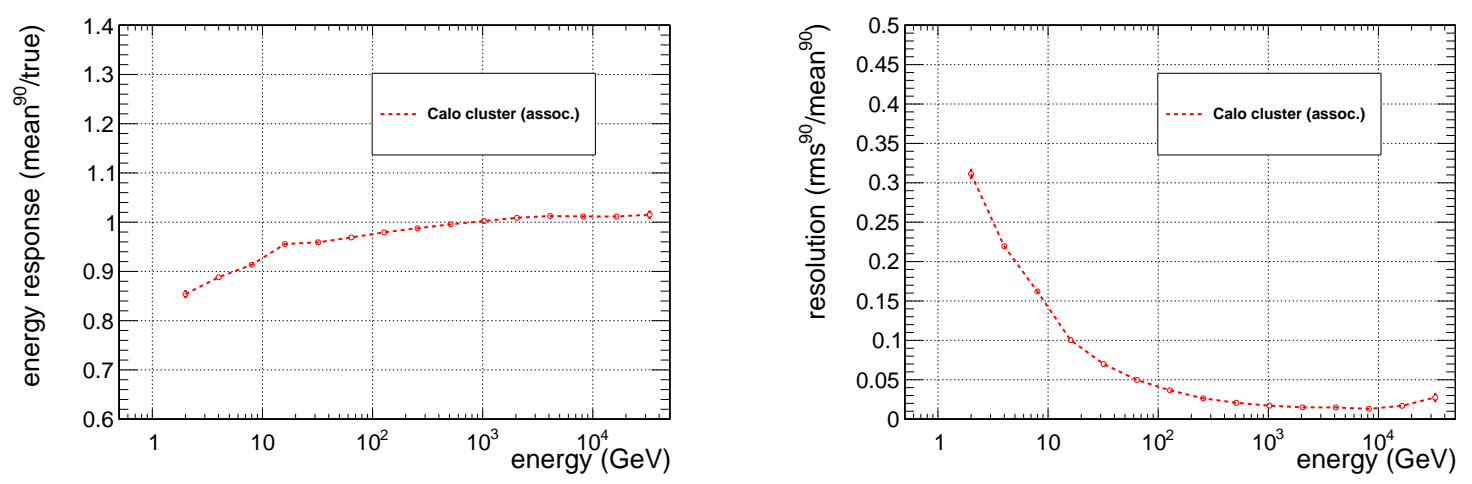

Figure 1: The calorimeter energy response (left) and resolution (right) of a single pion as a function of true pion energy.

\section{Weighted Energy Response}

We compare the calorimeter jet energy with the weighted and smeared energy of its corresponding generator-level jet. Within a jet, charged pions, photons, and charged kaons contribute 
$\approx 40 \%, 24 \%$, and $11 \%$ of the jet energy, respectively, while protons, neutrons, $\mathrm{K}_{s}^{0}$, and $\mathrm{K}_{L}^{0}$ contribute $\approx 5 \%$ each. The rest of the energy comes from baryons. Before clustering the generatorlevel particles with the anti-kt algorithm, we modify the energy of each generator-level particle with weighing and smearing. Figure 1 shows a loss of acceptance for low-momentum tracks due to the 5-Tesla magnetic field. Therefore, the response of all charged particles with energy below $2 \mathrm{GeV}$ is set to zero. The response of electrons and photons is set to unity and the energy is smeared using a resolution of $(0.15 / \sqrt{E} \oplus 0.01)$. Finally, the energies of all other charged particles are weighted and smeared following the response and resolution functions in Eqn. 4.1. Figure 2 shows the distributions of the ratio of weighted and smeared energy to the true jet energy from the $Z^{\prime}$ and the $\mathrm{H}_{2}$ samples; the true jet energy is defined as the generator-level jet energy before weighing and smearing (neutrinos are excluded in the clustering). The weighted and smeared distributions are narrower than the calorimeter energy distributions; in addition, the overall means of the former distributions are higher. This study indicates that the calorimeter jet energy response and resolution can not be modeled and extrapolated simply from those of the single-particle samples.
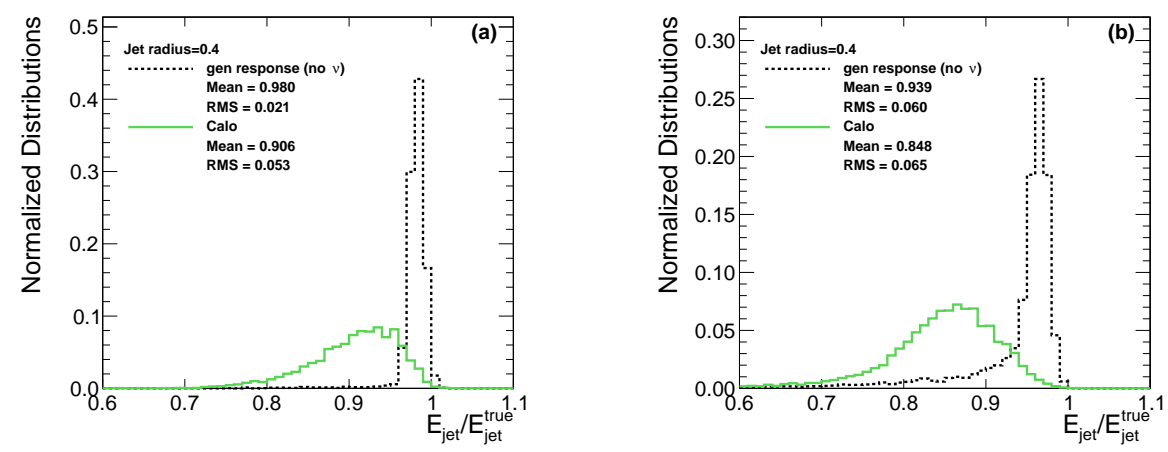

Figure 2: Ratio of the weighted and smeared generator-level jet energy to the true jet energy, from (a) W-jets in the $\mathrm{Z}^{\prime} \rightarrow \mathrm{WW}$ and from (b) Higgs-jets in the $\mathrm{H}_{2} \rightarrow$ hh samples. The masses of the $\mathrm{Z}^{\prime}$ and $\mathrm{H}_{2}$ resonances are $10 \mathrm{TeV}$ and $5 \mathrm{TeV}$, respectively. The weighted and smeared distributions (dashed lines) are compared with the distributions measured with the SiFCC calorimeters (solid lines).

\section{Energy Response and Resolution of W-jets}

We study the energy response and resolution of W-jets using the $\mathrm{Z}^{\prime} \rightarrow$ WW samples with $\mathrm{Z}^{\prime}$ mass at 5-40 TeV. The $|\eta|$ of $\mathrm{W}$-jets must be less than 1.1. Figure 3 shows the $90 \%$-truncated mean and RMS/mean of the jet energy ratios. The sampling term of the $\mathrm{W}$-jet energy resolution for these extreme energies is $\approx 237 \%$ while the constant term is $\approx 2.7 \%$; the sampling term is much higher than our expectation. However, note that this is the first time that the response and resolution of such high-energy jets have been studied with a full detector simulation. Currently, studies are ongoing to understand the reconstruction of calorimeter clusters used for jets.

\section{Two-Jet Separation In Boosted Bosons}

In addition to the energy response and resolution, we study the angular separation of two jets within highly boosted bosons. The angular separation is quantified by a variable, signed $\Delta R$, which 

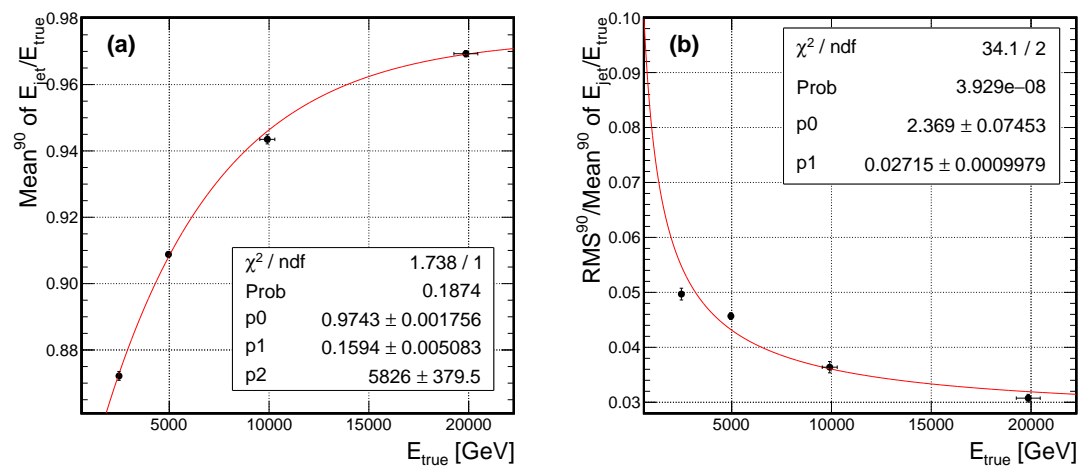

Figure 3: The (a) energy response and (b) resolution of the $\mathrm{W}$-jets from the $\mathrm{Z}^{\prime} \rightarrow \mathrm{WW}$ samples. The response is fitted with a function $y_{\text {response }}=p_{0}\left(1-p_{1} e^{-x / p_{2}}\right)$ while the resolution is fitted with a function $y_{\text {resolution }}=p_{0} / \sqrt{E_{\text {true }}} \oplus p_{1}$ as indicated by the curves.

is defined as the displacement of a calorimeter hit within a $\mathrm{W}$-jet (Higgs-jet), in the $\eta-\phi$ plane, with respect to the generator-level W (Higgs) boson-direction, projected onto the $\mathrm{q}-\overline{\mathrm{q}}$ axis $(\mathrm{b}-\overline{\mathrm{b}}$ )

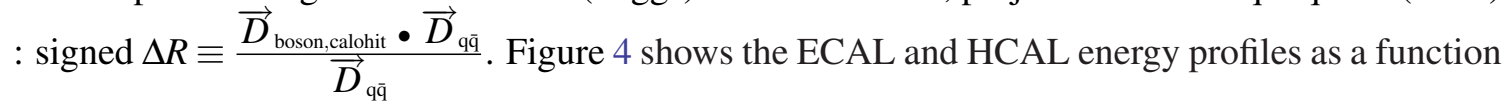
of signed $\Delta R$ for the $\mathrm{W}$-jets and Higgs-jets. The high-granularity calorimeter makes it possible to see the separation of subjets within highly-boosted W-jets and Higgs-jets.
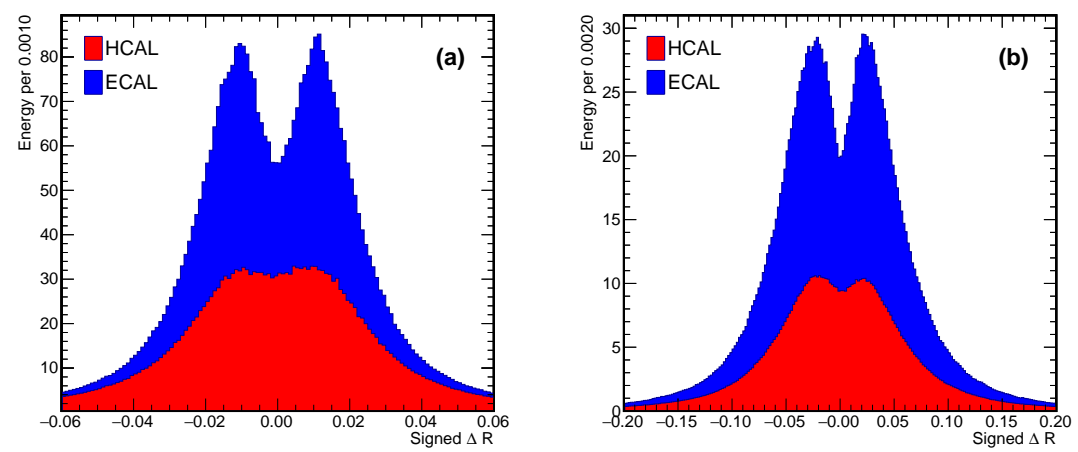

Figure 4: The ECAL and HCAL energy profiles as a function of signed $\Delta R$ from (a) $\mathrm{W}$-jets in the $\mathrm{Z}^{\prime} \rightarrow \mathrm{WW}$ and from (b) Higgs-jets in the $\mathrm{H}_{2} \rightarrow$ hh samples. The masses of the $\mathrm{Z}^{\prime}$ and $\mathrm{H}_{2}$ resonances are $10 \mathrm{TeV}$ and $5 \mathrm{TeV}$, respectively. The separation between the two peaks in each distribution is $\approx 0.02$ for $\mathrm{W}$-jets and $\approx 0.05$ for Higgs-jets.

\section{Conclusion}

In this document, we present a first study of single particle response and boosted $\mathrm{W}$-jets and Higgs-jets at the tens-TeV energy scale using a full GEANT4 simulation. The energy response and resolution of single particles follow the expected performance of the designed detector while the 
energy resolution of calorimeter jets is worse than the expected values. The high granularity of the SiFCC calorimeters shows the possibility to separate energy clusters within a jet from a 2.5-5 TeV boosted boson. We are geared towards the study of high-level variables related to jet substructure and techniques for jet grooming.

\section{Acknowledgments}

This research was done using resources provided by the Open Science Grid, which is supported by the National Science Foundation and the U.S. Department of Energy's Office of Science. The work of SC was supported by the U.S. Department of Energy, Office of Science under Contract No. DE-AC02-06CH11357. The work of AVK was supported by the Fermi National Accelerator Laboratory. Fermilab is operated by Fermi Research Alliance, LLC, under Contract No. DEAC02-07CH11359 with the United States Department of Energy.

\section{References}

[1] A. Ball et al., "Future Circular Collider Study - Hadron Collider Parameters," FCC-ACC-SPC-0001 rev. 1.0 (2014).

[2] J. Tang et al., "Concept for a Future Super Proton-Proton Collider," arXiv:1507.03224.

[3] CEPC-SPPC Study Group, “CEPC-SPPC Preliminary Conceptual Design Report. 2. Accelerator," IHEP-CEPC-DR-2015-01, IHEP-AC-2015-01.

[4] T. Carli, C. Helsens, A. Henriques Correia and C. Solans SÃąnchez, "Containment and resolution of hadronic showers at the FCC," JINST 11, no. 09, P09012 (2016).

[5] H. Aihara et al., "SiD Letter of Intent," arXiv:0911.0006.

[6] C. Adolphsen et al., "The International Linear Collider Technical Design Report - Volume 3.II: Accelerator Baseline Design," arXiv:1306.6328.

[7] T. Behnke et al., "The International Linear Collider Technical Design Report - Volume 4: Detectors," arXiv:1306.6329.

[8] S. V. Chekanov, K. Strand and P. Van Gemmeren, "ProMC: Input-output data format for HEP applications using varint encoding," Comput. Phys. Commun. 185, 2629 (2014).

[9] T. Sjostrand, S. Mrenna and P. Z. Skands, "PYTHIA 6.4 Physics and Manual,” JHEP 0605, 026 (2006).

[10] J. Alwall et al., "The automated computation of tree-level and next-to-leading order differential cross sections, and their matching to parton shower simulations," JHEP 1407, 079 (2014).

[11] S. Agostinelli et al., "GEANT4: A Simulation toolkit," Nucl. Instrum. Meth. A 506, 250 (2003).

[12] S. V. Chekanov, "HepSim: a repository with predictions for high-energy physics experiments," Adv. High Energy Phys. 2015, 136093 (2015).

[13] R. Pordes et al., J. Phys. Conf. Ser. 78, 012057 (2007).

[14] M. Cacciari, G. P. Salam and G. Soyez, "The Anti-k(t) jet clustering algorithm," JHEP 0804, 063 (2008).

[15] M. Cacciari, G. P. Salam and G. Soyez, “FastJet User Manual,” Eur. Phys. J. C 72, 1896 (2012). 